\title{
Anomalous reflection of gold: a novel platform for biochips
}

\begin{abstract}
The importance of protein detection system for protein functions analyses in recent postgenomic era is rising with the emergence of label-free protein detection methods. We are focusing on a simple and practical label-free optical-detection method called anomalous reflection (AR) of gold. When a molecular layer forms on the gold surface, significant reduction in reflectivity can be observed at wavelengths of 400-500 $\mathrm{nm}$. This allows the detection of molecular interactions by monitoring changes in reflectivity. In this chapter, we describe the AR method with three different application platforms: (1) gold, (2) gold containing alloy/composite (AuAg2O), and (3) metal-insulator-metal (MIM) thin layers. The $\mathrm{AuAg} 2 \mathrm{O}$ composite and MIM are implemented as important concepts for signal enhancement process for the AR technique. Moreover, the observed molecular adsorption and activity is aided by a three-dimensional surface geometry, performed using poly(amidoamine) or PAMAM dendrimer modification. The described system is suitable to be used as a platform for high-throughput detection system in a chip format.
\end{abstract}

Keywords: Protein-peptide interactions; Label-free detection method; Anomalous reflection (AR) of gold; Gold platform; Surface chemistry; Glass substrate 